\title{
(a)
}

\section{Biomarkers associated with cardiovascular events in CKD}

The biomarkers of cardiac damage, high-sensitive troponin $\mathrm{T}$ (hsTnT) and $\mathrm{N}$-terminal pro-B-type natriuretic peptide (NT-pro-BNP), are prognostic markers for cardiovascular events in patients with chronic kidney disease (CKD) and not merely a reflection of reduced renal clearance, show investigators from The Netherlands. "It is always argued that increased hsTnT and NT-pro-BNP levels are not reliable markers for cardiac damage in CKD owing to the fact that they are spuriously elevated", explains researcher Paul de Jong. "We questioned that statement and wanted to show that both parameters in CKD are still related to cardiac survival also after adjustment for glomerular filtration rate (GFR) and albuminuria."

Among 8,121 individuals in the PREVEND study with available hsTnT and NT-pro-BNP values, 6.7\% had increased hsTnT levels and $12.2 \%$ had an elevated NT-pro-BNP concentration. Patients with increased levels of these biomarkers had a worse cardiovascular risk profile with more ischaemic changes and left ventricular hypertrophy as assessed by electrocardiography. These biomarkers were also significantly associated with a lower estimated GFR and higher

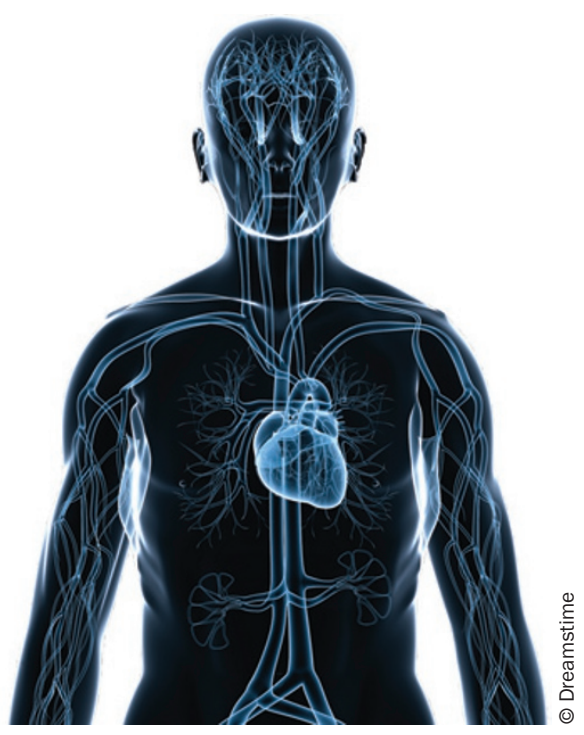

in patients with CKD in adjusted analyses. As only a small number of individuals included in the population had markedly reduced renal function, the results point to cardiac damage already early in CKD. As de Jong points out, "these parameters truly reflect cardiac damage in early stages of CKD and they may help to unravel the cause of the association of CKD with cardiovascular end points."

Elevated hsTnT and NT-pro-BNP levels are assumed to be associated with ischaemia and left ventricular hypertrophy, respectively, but these assumptions might not be correct. "The association of both parameters with cardiovascular events was still significant after adjusting for ischaemic changes and left ventricular hypertrophy", says de Jong. "The increased levels of these cardiac enzymes may thus point to a specific CKD-related pathology, which should be studied in the future."

Helene Myrvang risk of cardiovascular events in patients with and without CKD in unadjusted and adjusted analyses. An increased level of hsTnT was associated with a higher risk of cardiovascular events in patients with and without CKD in unadjusted analyses, but was only associated with an increased risk
Original article Scheven, L. et al. High-sensitive troponin T and $\mathrm{N}$-terminal pro-B type natriuretic peptide are associated with cardiovascular events despite the crosssectional association with albuminuria and glomerular filtration rate. Eur. Heart J. doi:10.1093/eurheartj/ehs163 